\title{
Evaluation of ustekinumab trough levels during induction and maintenance therapy with regard to disease activity status in difficult to treat Crohn disease patients
}

\author{
Nicolae-Catalin Mechie, $\mathrm{MD}^{\mathrm{a}}$, Merle Burmester ${ }^{\mathrm{a}}$, Eirini Mavropoulou, MDª ${ }^{\mathrm{a}}$, Yiannis Pilavakis, MD ${ }^{\mathrm{b}}$, \\ Steffen Kunsch, MD ${ }^{a}$, Volker Ellenrieder, MDa , Ahmad Amanzada, MD ${ }^{a}{ }^{*}$
}

\begin{abstract}
Ustekinumab (UST) is approved for the treatment of moderate and severe Crohn disease (CD). Therapeutic drug monitoring (TDM) can help monitor the therapeutic effects of biologics. Therefore, the aim of this study was to evaluate the clinical outcomes of USTtreated CD patients and to determine the UST trough level in clinical and corticosteroid-free remission.

This retrospective study included patients with moderate and severe active disease (AD) treated intravenously with a weightadapted induction dose of UST. The maintenance therapy consisted of $90 \mathrm{mg}$ UST subcutaneously at week 8 and thereafter every 8 or 12 weeks, depending on the clinical response. Clinical and corticosteroid-free remission, Harvey-Bradshaw-Index (HBI), UST trough level, and further laboratory parameters were measured just before the injection of UST at each follow-up evaluation until week 40 .

$37 \mathrm{CD}$ patients with a median $\mathrm{HBI}$ of 9 at week 0 were included in the study. Starting from $24 \%$ at the beginning of the monitoring period, and $38 \%$ of patients at the end of the monitoring period were treated with an 8 -week interval $(P=.18)$. There was a significant improvement in clinical $(P=.0004)$, corticosteroid-free remission $(P=.03)$, and $\mathrm{HBI}(P<.0001)$ from week 0 until the end of the observation period. The serum UST trough level decreased significantly from 2.0 at week 8 to 0.3 , in the maintenance therapy and $0.4 \mu \mathrm{g} / \mathrm{ml}$ at the end of the therapy $(P<.0001)$. Neither UST trough level nor levels of C-reactive protein (CRP) or fecal calprotectin (FC) were associated with disease outcome. Concomitant immunomodulator therapy did not appear to affect the UST trough level or clinical course.

UST is an effective treatment option for difficult-to-treat patients with CD. UST trough levels may not be associated with treatment efficacy or the prediction of treatment outcomes in patients with CD. Further prospective randomized trials should be conducted to evaluate whether UST trough levels are associated with treatment outcomes in patients with CD.

Abbreviations: $A=$ age,$A D=$ active disease, $C D=$ Crohn disease, $C R=$ clinical remission, $C R P=C$-reactive protein, $E L I S A=$ enzyme-linked immunosorbent assay, $\mathrm{FC}=$ Fecal calprotectin, $\mathrm{HBI}=$ Harvey-Bradshaw-Index, IBD = inflammatory bowel disease, $\mathrm{IL}=$ interleukin, $\mathrm{IQR}=$ interquartile range, $\mathrm{TO}=$ time of induction, $\mathrm{T} 1=8$ weeks after induction, $\mathrm{T} 2=16-20$ weeks after induction, $\mathrm{T} 3$ $=24-28$ weeks after induction, T4 $=32-40$ weeks after induction, TDM = therapeutic drug monitoring, TNF- $\alpha=$ tumor necrosis factor alpha, UST = ustekinumab.
\end{abstract}

Keywords: Crohn disease, therapeutic drug monitoring, ustekinumab

\footnotetext{
Editor: Yang Cheng.

This work was supported by the Open Access Publication Funds of Göttingen University.

The authors have no conflicts of interest to disclose.

The datasets generated during and/or analyzed during the current study are available from the corresponding author on reasonable request.

${ }^{a}$ Clinic for Gastroenterology, Gastrointestinal Oncology and Endocrinology, ${ }^{b}$ Clinic of Otorhinolaryngology, Head and Neck Surgery, University Medical Center Goettingen, Germany.

* Correspondence: Ahmad Amanzada, Clinic for Gastroenterology, Gastrointestinal Oncology and Endocrinology, University Medical Center Goettingen, Robert-KochStr. 40, Goettingen 37075, Germany (e-mail: ahmad.amanzada@med.uni-goettingen.de).

Copyright (c) 2021 the Author(s). Published by Wolters Kluwer Health, Inc.

This is an open access article distributed under the terms of the Creative Commons Attribution-Non Commercial License 4.0 (CCBY-NC), where it is permissible to download, share, remix, transform, and buildup the work provided it is properly cited. The work cannot be used commercially without permission from the journal.

How to cite this article: Mechie NC, Burmester M, Mavropoulou E, Pilavakis Y, Kunsch S, Ellenrieder V, Amanzada A. Evaluation of ustekinumab trough levels during induction and maintenance therapy with regard to disease activity status in difficult to treat Crohn disease patients. Medicine 2021;100:11(e25111).

Received: 19 October 2020 / Received in final form: 14 February 2021 / Accepted: 18 February 2021

http://dx.doi.org/10.1097/MD.0000000000025111
} 


\section{Introduction}

Crohn's disease (CD) is an inflammatory bowel disease (IBD) characterized by transmural segmental inflammation of the gastrointestinal tract. The clinical course of CD involves chronic inflammation resulting in abdominal pain, chronic diarrhea, gastrointestinal bleeding, and complications, including the development of strictures and fistulas. To date, the pathogenesis of $\mathrm{CD}$ remains unclear, and there is currently no cure for $\mathrm{CD}$. The treatment consists of administering immunomodulating therapy, such as corticosteroids, immunosuppressants, and biological agents, such as tumor necrosis factor alpha (TNF- $\alpha$ ) antagonists, integrin, and interleukin (IL) inhibitors. ${ }^{[1]}$ Unfortunately, surgery has no curative effect on $\mathrm{CD}$, and most patients require treatment with different therapies during their lifetime. The use of biological agents has revolutionized CD therapy. ${ }^{[2]}$ Ustekinumab (UST) is a biologic agent approved for the treatment of moderate and severe $\mathrm{CD}$, even in patients with a previous history of anti-TNF- $\alpha$ treatment. UST inhibits the p40 subunit of interleukin (IL)12 and IL23. ${ }^{[3]}$ In clinical care, the majority of patients with CD have already been exposed to anti-TNF- $\alpha$ or other immunomodulatory therapies.

Therapeutic drug monitoring (TDM) is an important key player in current IBD therapy and is recommended when using anti-TNF- $\alpha$ agents, such as adalimumab and infliximab. ${ }^{[4]}$ TDM can be conducted in 2 ways: the first is proactive, in which the TDM is made even in the absence of IBD-related symptoms. The other is reactive, where TDM is determined only when the patient has a flair of IBD. ${ }^{[4]}$ Existing data regarding the UST and TDM have been the result of highly selected patient groups from approval studies or from studies where the observational time was short. ${ }^{[5]}$

Because of the scarce data regarding TDM and partially conflicting results between the data from the approval and reallife studies, we decided to investigate the association of serum UST levels with clinical and corticosteroid-free remission in CD patients after induction and maintenance therapy.

\section{Methods}

\subsection{Study population}

We retrospectively assessed a prospectively maintained database of patients with CD who were treated at our center. Our cohort consisted of 37 consecutive patients with CD who received UST at the Goettingen University Medical Center. Furthermore, patients were included only if they had an available UST trough level at week 8 after induction therapy and a second measurement during maintenance therapy. The Harvey-Bradshaw index (HBI) was used for the clinical assessment of CD. Clinical remission (CR) was defined as $\mathrm{HBI}<5$ points. Corticosteroid-free remission was defined as achieving $\mathrm{CR}$ with complete withdrawal of steroids in patients who had previously received cortisone therapy at the time of UST induction.

The following biochemical parameters were analyzed: hemoglobin level, platelet and leukocyte count, C-reactive protein (CRP) level (normal $<5 \mathrm{mg} / \mathrm{L}$ ), fecal calprotectin (FC) level (normal $<50 \mathrm{mg} / \mathrm{kg}$ ), and UST trough levels in serum. Blood laboratory values were determined immediately before administration of UST.

UST was administered according to an approved protocol. Induction was performed with intravenous, weight-adapted doses (less than $55 \mathrm{~kg} 260 \mathrm{mg}$, between $55 \mathrm{~kg}$ and $85 \mathrm{~kg} 390 \mathrm{mg}$, and more than $85 \mathrm{~kg} 520 \mathrm{mg}$ ). The maintenance therapy was performed by administering $90 \mathrm{mg}$ UST subcutaneously at week 8 after the induction therapy and thereafter every 8 or 12 weeks, depending on the clinical response. The UST interval was shortened only in cases of clinical disease activity. The following treatment intervals were defined: T0 (time of induction), T1 (8 weeks after induction), T2 (16-20 weeks after induction), T3 (24-28 weeks after induction), and T4 (32-40weeks after induction). Induction and maintenance of UST therapy were administered exclusively by the medical staff at the university medical center. Weeks 32 to 40 were chosen to assess the outcome of UST induction and maintenance because they also included patients with delayed clinical response to UST.

Serum concentrations of CRP, hemoglobin, platelets, and leukocyte counts were determined using the automated systems of the Central Laboratory of the Department of Clinical Chemistry at the University Medical Center Goettingen. FC levels were measured as previously described. ${ }^{[6]}$

UST was quantified in serum samples using an enzyme immunoassay ((IDK monitor UST drug level enzyme-linked immunosorbent assay (ELISA), immundiagnostik AG, Germany)) according to the manufacturer's recommendations. The lowest quantifiable concentration in a sample for this method was $0.3 \mu \mathrm{g} / \mathrm{ml}$. Serum UST levels were measured in an external laboratory. The determination method was established and validated as a routine procedure.

The datasets analyzed in the current study are available from the corresponding author upon reasonable request.

\subsection{Ethic statement}

This study was approved by the local ethics committee (approval number 7/10/19).

\subsection{Statistical analysis}

Continuous variables are expressed as median and interquartile range (IQR), and categorical variables are expressed as percentages. For comparisons of variables, a nonparametric 1way analysis of variance based on the median score was used for continuous and ordinal variables, whereas Fisher exact test was used for categorical variables. Statistical significance was set at $P<.05$. We further performed Spearman correlation analysis between the continuous variables. Statistical analyses were performed using the SPSS 26 software for Mac OS (SPSS, Chicago, IL) and GraphPad Prism 5.

\section{Results}

Of the 37 patients with CD at the time of induction, 25 were men with a median disease duration of 15 years. The majority of patients were between 17 and 40 years of age at the time of the first diagnosis of CD. Most patients had ileocolonic, penetrating, or perineal involvement. At UST induction, $51 \%$ of patients had corticosteroids, $14 \%$ azathioprine, and $30 \%$ tacrolimus as concomitant therapy. $57 \%$ of CD patients were under monotherapy with azathioprine, tacrolimus, or corticosteroids at the time of induction with UST. Six, 24, and 7 patients received UST induction doses of 260, 390, or $520 \mathrm{mg}$, respectively. All patients were previously exposed to a TNF- $\alpha$ antagonist (Table 1). Following parameters were recorded at the time of induction therapy: the median HBI was 9 and the median serum albumin 


\section{Table 1}

Patients' demographic and clinical characteristics.

\begin{tabular}{|c|c|}
\hline & Total $(n=37)$ \\
\hline Median Age, year (IQR) & $48(33-55)$ \\
\hline Female / Male, n (\%) & $25 / 12$ (68/32) \\
\hline Median disease duration, year (IQR) & $15(7-22)$ \\
\hline $\begin{array}{l}\text { Montreal age at diagnosis of } \\
\text { CD: A1 / A2 / A3, n (\%) }\end{array}$ & 10 / 23 / 4 (27/62/11) \\
\hline $\begin{array}{l}\text { Montreal disease behavior of } \\
\text { CD: B1 / B2 / B3 / B3p, n (\%) }\end{array}$ & $10 / 6 / 6 / 15(27 / 16 / 16 / 41$ \\
\hline $\begin{array}{l}\text { Montreal localization of } \\
\text { CD: L1 / L2 / L3 / L4, n (\%) }\end{array}$ & $7 / 4 / 21 / 5$ (19/11/57/13) \\
\hline \multicolumn{2}{|l|}{ Medication profile, $\mathrm{n}(\%)$} \\
\hline Steroids & $19(51)$ \\
\hline Azathioprine & $5(14)$ \\
\hline Tacrolimus & $11(30)$ \\
\hline \multicolumn{2}{|l|}{ Therapy, n (\%) } \\
\hline Monotherapy & $21(57)$ \\
\hline Combinationtherapy & $16(43)$ \\
\hline Endoscopic activity ${ }^{*}, \mathrm{n}(\%)$ & $25(68)$ \\
\hline \multicolumn{2}{|l|}{ Initial Ustekinumab dose, $\mathrm{n}(\%)$} \\
\hline $260 \mathrm{mg}$ & $6(16)$ \\
\hline $390 \mathrm{mg}$ & $24(65)$ \\
\hline $520 \mathrm{mg}$ & 7 (19) \\
\hline History of TNF- $\alpha$ antagonist use, $n(\%)$ & $37(100)$ \\
\hline
\end{tabular}

$A=$ age, $B=$ behavior, $C D=$ Crohn disease, IQR = interquartile range, $L=$ localization, $T N F-\alpha=$ tumor necrosis factor alpha.

*25 patients with available endoscopic evaluation.

level was $3.5 \mathrm{~g} / \mathrm{dl}$, the median platelet and leukocyte count were $313 \times 10^{3} / \mu \mathrm{l}$ and $8.5 \times 10^{3} / \mu \mathrm{l}$, respectively, as well as the median serum CRP level was $6.9 \mathrm{mg} / \mathrm{L}$ and median FC level $458 \mathrm{mg} / \mathrm{kg}$ (Table 2).

At the time of induction therapy, $11 \%$ of the patients were in CR. All patients received therapy consisting of an immunomodulator or corticosteroid. Moreover, 3 of these patients showed a corticosteroid-dependent course. The rate of CR increased significantly during maintenance therapy from $41 \%$ at $\mathrm{T} 1$ to $54 \%$ at T4 $(P=.0004)$ (Fig. 1A). The rate of corticosteroid-free remission at the time of induction was $6 \%$, which increased significantly during maintenance therapy to $33 \%$ at T4 $(P=.03)$ (Fig. 1B).

During maintenance therapy, the median HBI decreased significantly from 9 at the time of induction to a median HBI of 5 between T1 and T3 and at the end of the study to a median HBI of 4 at T4 $(P<.0001)$ (Table 2$)$.
Repeated evaluation of serum albumin level $(P<.19)$, platelet count $(P<.93)$, leukocyte count $(P<.68)$, CRP $(P<.91)$, and FC $(P<.32)$ levels did not change over time (Table 2$)$. Repeated measurement of median serum UST trough level decreased significantly after the intravenous induction dose from 2.0 to 0.3 , 0.3 , and 0.4 , at T2, T3, and T4 $(P<.0001)$, respectively. There was no significant change in the rate of combination therapy $(P<.75)$ or the application interval of UST therapy $(P<.18)$ (Table 2).

The median serum UST trough level at T1 was 1.9 and $2.0 \mu \mathrm{g} /$ $\mathrm{ml}$ in patients with $\mathrm{CR}$ and active disease (AD), respectively $(P=.57)$. In patients who achieved a CR and those with AD the median serum UST trough levels decreased during maintenance therapy to 0.3 and $0.3 \mu \mathrm{g} / \mathrm{ml}$ at T2 $(P=.90), 0.3$ and $0.3 \mu \mathrm{g} / \mathrm{ml}$ at T3 $(P=.76)$ as well as to 0.4 and $0.5 \mu \mathrm{g} / \mathrm{ml}$ at T4 $(P=.75)$, respectively. The UST trough level measured at T1 did not predict CR to induction therapy for T2 $(P=.54)$, T3 $(P=.15)$, or T4 $(P=.58)$, nor was it associated with corticosteroid-free remission at T1 $(P=.05)$, T2 $(P=.23)$, T3 $(P=.31)$, or T4 $(P=.40)$, respectively. A nonparametric 1 -way analysis of variance showed no association with disease activity status for median serum UST trough level $(P=.88$, Fig. 2A), CRP levels $(P=.69$, Fig. $2 \mathrm{~B})$, and FC levels $(P=.53$, Fig. 2C).

Finally, we performed Spearman correlation between serum UST trough levels and serum albumin, platelet and leukocyte counts, and CRP and FC levels. There were no correlations between these parameters (data not shown).

\section{Discussion}

The proinflammatory cytokines IL12 and IL23 have been implicated in the pathophysiology of $\mathrm{CD}$, with multiple lines of evidence suggesting that CD is mediated by T-helper 1 and/or T-helper 17 cells. ${ }^{[7,8]}$ UST prevents IL12 and IL23 bioactivity by preventing their interaction with the cell surface receptor protein IL12Rb1. Through this mechanism of action, UST effectively neutralizes IL12 (T-helper 1) and IL23 (T-helper 17) mediated cellular responses. UST binds with high affinity to the $\mathrm{p} 40$ subunit of human IL12 and IL23 and has recently been approved for the treatment of moderately to severely active CD in adults.

Currently, UST is an effective treatment for patients with antiTNF- $\alpha$ refractory CD. It has been shown to be useful in the management of patients with a loss of response to anti-TNF- $\alpha$ agents. However, data on the association between UST drug concentrations and CD patient outcomes are limited. Understanding UST pharmacokinetic characteristics and the relation-

\section{Table 2}

Comparison of patient characteristics between induction and maintenance therapy.

\begin{tabular}{|c|c|c|c|c|c|c|}
\hline & TO & T1 & T2 & T3 & T4 & $P$ Value \\
\hline Median HBI, (IQR) & $9(8-12)$ & $5(2-7)$ & $5(2-10)$ & $5(2-8)$ & $4(3-8)$ & $<0.0001$ \\
\hline Median Albumin level, g/dl (IQR) & $3.5(3.1-3.7)$ & $3.7(3.4-3.9)$ & $3.7(3.4-3.9)$ & $3.7(3.4-3.9)$ & $3.7(3.4-3.9)$ & 0.19 \\
\hline Median platelet count, $10^{3} / \mu \mathrm{l}(\mathrm{IQR})$ & $313(227-436)$ & $306(233-385)$ & $318(248-380)$ & $306(261-388)$ & $300(239-361)$ & 0.93 \\
\hline Median CRP level, mg/L (IQR) & $6.9(2.8-14.6)$ & $5.4(1.8-15.1)$ & $5.7(2.3-19.6)$ & $6.2(2.5-17.9)$ & $6.8(3.0-17.6)$ & 0.91 \\
\hline Median FC level, mg/kg (IQR) & 458 (347-702) & $225(74-448)$ & 391 (126-1032) & $283(66-444)$ & 267 (96-479) & 0.32 \\
\hline Median UST trough level, $\mu \mathrm{g} / \mathrm{ml}$ (IQR) & NA & $2.0(1.2-4.0)$ & $0.3(0.3-0.4)$ & $0.3(0.3-0.6)$ & $0.4(0.3-0.8)$ & $<0.0001$ \\
\hline
\end{tabular}

$\mathrm{CRP}=\mathrm{C}$-reactive protein, $\mathrm{FC}=$ fecal calprotectin, $\mathrm{HBI}=$ Harvey-Bradshaw-Index, IQR = interquartile range, $\mathrm{T} 0=$ time of induction, $\mathrm{T} 18$ weeks after induction, $\mathrm{T} 2=16-20$ weeks after induction, $\mathrm{T} 3=24-28$ weeks after induction, T4 = 32-40 weeks after induction, UST = Ustekinumab. 

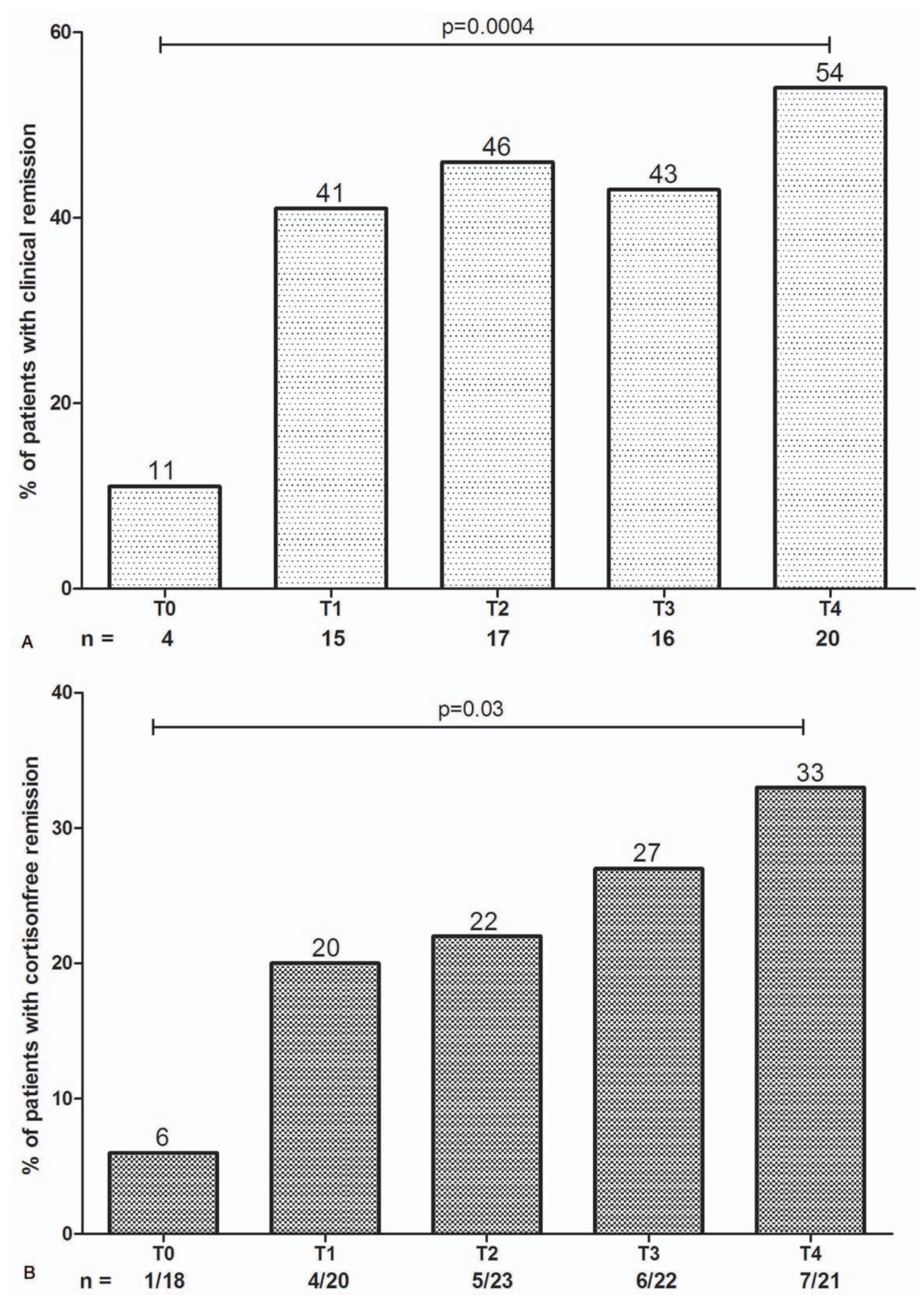

Figure 1. Clinical (A) and corticosteroid-free (B) outcomes in Crohn disease patients receiving ustekinumab. TO (time of induction), T1 (8 weeks after induction), T2 (16-20 weeks after induction), T3 (24-28weeks after induction) and T4 (32-40weeks after induction).

ship between clinical outcomes and UST concentration is important for prescribers to optimize the efficacy of UST therapy.

The main finding of this study is that anti-TNF- $\alpha$ refractory CD patients treated with UST achieved in $54 \%$ and $33 \%$ of cases clinical and corticosteroid-free remission over a treatment period of 32 to 40 weeks. In addition, the UST trough level decreased significantly during maintenance therapy, just above the detection limit. The mean UST concentration at week 8 in our 

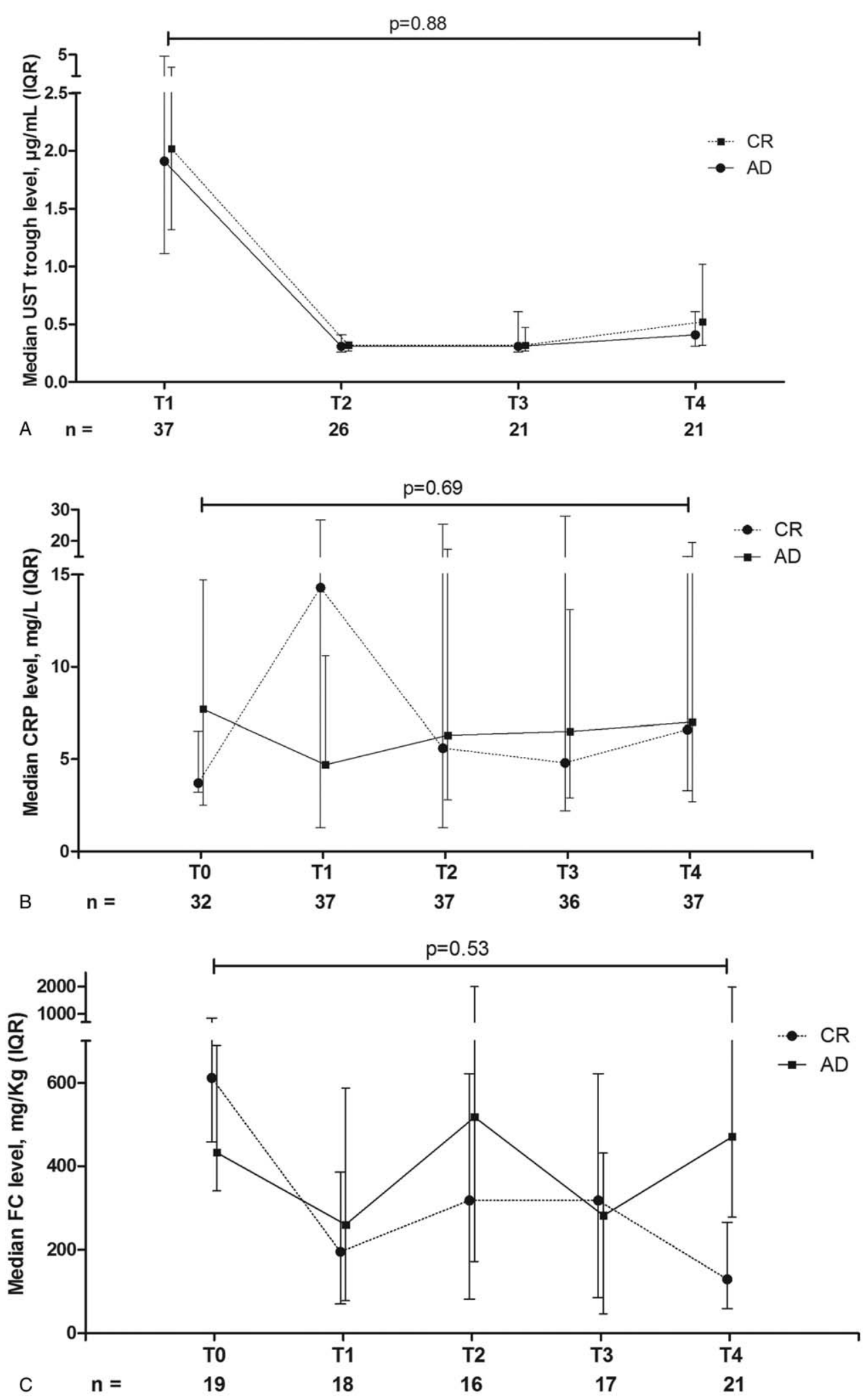

Figure 2. Association of clinical disease status (CR: clinical remission, AD: active disease) with regard to ustekinumab (UST) trough levels (A), $C$ reactive protein $(\mathrm{CRP})(\mathrm{B})$ and fecal calprotectin $(\mathrm{FC})(\mathrm{C})$ level with the interquartile range (IQR).

study was similar to that reported by Adedokun et al: 2.0 (IQR $1.2-4.0 \mu \mathrm{g} / \mathrm{ml}) .^{[5]}$ Interestingly, the present real-life study does not report a positive relationship between UST trough levels and clinical disease outcomes during UST therapy. In addition, the levels of proinflammatory biochemical parameters, such as CRP and FC, neither decreased during the treatment period nor were 
associated with treatment outcome. The majority of the included patients were treated with a 12 -week interval during maintenance therapy.

Our findings show a discrepancy from those found in previous studies in which a UST trough level of $>4.5 \mu \mathrm{g} / \mathrm{ml}$ at week 26 was associated with endoscopic response ${ }^{[9]}$ or a trough level of $3.3 \mu \mathrm{g} /$ $\mathrm{ml}$ at week 8 was associated with CR. ${ }^{[5]}$ In line with our results, the study by Battat et al also failed to identify an association between UST trough levels and clinical outcomes. ${ }^{[9]}$ Another study by Thomann et al also identified that serum UST level at week 8 was moderately effective in predicting clinical response at week 16. ${ }^{[10]}$ Recently, Hanzel et al showed that UST concentrations above $105 \mu \mathrm{g} / \mathrm{ml}$ measured one hour after induction were associated with endoscopic remission in CD patients. ${ }^{[11]}$

The pharmacokinetics post hoc analysis of the approval studies of UST published by Adedokun et al showed that during the maintenance study period, UST concentration reached a steady state after the second maintenance dose. Moreover, the median trough concentration in patients administered UST every 8-weeks compared to patients every 12 -weeks was approximately threefold higher. ${ }^{[5]}$ UST serum concentrations were significantly associated with rates of CR only in patients treated with an 8week interval $(P=.006)$ but not with a 12-week interval application $(P=.08)$. In contrast to the results of the present study, they could show that trough concentrations of UST of $0.8 \mu \mathrm{g} / \mathrm{ml}$ or greater were associated with maintenance of CR. In addition, the UST concentration cut offs obtained from the receiver operator curve analyses were based on statistically significant but modest areas under the curve and specificity values. ${ }^{[5]}$ Because the level of significance could only be achieved in the 8 -week interval treatment, this result has no general validity in a real-life setting, as the majority of patients may be treated with a 12 -week interval.

In accordance with our results, Peinchart et al showed, by studying 49 patients who received maintenance therapy, that UST trough level did not correlate with clinical, biological, or endoscopic response. ${ }^{[12]}$

Various factors may account for such differences, including distinct treatment regimens and disease outcome assessments, different assays for measuring UST, and distinct patient populations. Only studies by Adedokan et al, Thomann et al, Soufflet et al, and our study evaluated CD patients after intravenous induction and subcutaneous maintenance therapy ${ }^{[5,10,13]}$ while other published studies evaluated different modes of induction and maintenance therapy. ${ }^{[9,12]}$

Different assay techniques are available on the market to determine the UST drug concentration, and the above-mentioned studies did not use the same assay to determine the UST cut offs, which may explain the different results obtained. The assays in previous studies used laboratory techniques such as ELISA, a drug-tolerant liquid-phase homogeneous mobility shift assay, or an electro chemiluminescent immunoassay. ${ }^{[5,9,12]}$ In this study, we used an ELISA assay. Thus, other prospective studies are needed to confirm the therapeutic UST trough levels.

In our cohort, all patients were previously treated or were intolerant to anti-TNF- $\alpha$. Anti-TNF- $\alpha$ therapy was changed due to anaphylaxis, primary and secondary nonresponse, or side effects. In the UST-approval studies, a medical history of antiTNF- $\alpha$ therapy was associated with lower rates of CR. ${ }^{[3]}$ The fact that we have only included patients exposed to anti-TNF- $\alpha$ in our study with a high inflammatory burden and complex pathophysiology could explain the contrasting results of our study.
In contrast to the experience with anti-TNF- $\alpha$ there was no significant impact of immunomodulators such as azathioprine or tacrolimus on serum UST concentration in the present study as well as previously published. ${ }^{[5,9]}$

We acknowledge the limitations of our study, such as its retrospective nature, the small number of patients in our cohort, and the fact that not all patients received endoscopic observation or FC monitoring during the analyzed period. Due to the small number of patients, we could not calculate the UST trough level for the group of patients treated with an 8-week interval compared to the 12 -week interval. However, we strongly believe that our results provide important clinical information, which could potentially help gastroenterologists treat CD with UST to make important decisions.

In conclusion, UST is a valid and helpful agent for the treatment of difficult-to-treat patients with CD. In our cohort, serum UST trough levels did not correlate with clinical or corticosteroid-free remission, with UST trough levels ranging just above the detection limit during maintenance therapy. Further large-scale real-life studies are needed to clarify the value of UST TDM for the treatment of CD.

\section{Author contributions}

Conceptualization: Nicolae-Catalin Mechie, Volker Ellenrieder, Ahmad Amanzada.

Data curation: Nicolae-Catalin Mechie, Merle Burmester, Eirini Mavropoulou, Steffen Kunsch, Ahmad Amanzada.

Formal analysis: Merle Burmester, Yiannis Pilavakis, Steffen Kunsch, Ahmad Amanzada.

Methodology: Nicolae-Catalin Mechie, Eirini Mavropoulou, Volker Ellenrieder, Ahmad Amanzada.

Resources: Merle Burmester.

Software: Merle Burmester, Steffen Kunsch.

Supervision: Steffen Kunsch, Volker Ellenrieder, Ahmad Amanzada.

Validation: Yiannis Pilavakis, Ahmad Amanzada.

Writing - original draft: Nicolae-Catalin Mechie, Merle Burmester, Ahmad Amanzada.

Writing - review \& editing: Eirini Mavropoulou, Yiannis Pilavakis, Volker Ellenrieder, Ahmad Amanzada.

\section{References}

[1] Torres J, Mehandru S, Colombel J-F, et al. Crohn's disease. Lancet 2017;3898:1741-55.

[2] Cholapranee A, Hazlewood GS, Kaplan GG, et al. Systematic review with meta-analysis: comparative efficacy of biologics for induction and maintenance of mucosal healing in Crohn's disease and ulcerative colitis controlled trials. Aliment Pharmacol Ther 2017;45:1291-302.

[3] Feagan BG, Sandborn WJ, Gasink C, et al. Ustekinumab as induction and maintenance therapy for Crohn's disease. N Engl J Med 2016;375: 1946-60.

[4] Casteele NV, Herfarth H, Katz J, et al. American gastroenterological association institute technical review on the role of therapeutic drug monitoring in the management of inflammatory bowel diseases. Gastroenterology 2017;153:835-57.

[5] Adedokun OJ, Xu Z, Gasink C, et al. Pharmacokinetics and exposure response relationships of ustekinumab in patients with Crohn's disease. Gastroenterology 2018;154:1660-71.

[6] Bathe AL, Mavropoulou E, Mechie N-C, et al. Plos One 2019;14: e0223893.

[7] Neurath MF. IL-23: a master regulator in Crohn disease. Nat Med 2007;13:26-8.

[8] Iwakura Y, Ishigame H. The IL-23/IL-17 axis in inflammation. J Clin Invest 2006;116:1218-22. 
[9] Battat R, Kopylov U, Bessissow T, et al. Association between ustekinumab trough concentrations and clinical, biomarker, and endoscopic outcomes in patients with Crohn's disease. Clin Gastroenterol Hepatol 2017;15:1427-34.

[10] Thomann AK, Schulte L-A, Globig A-M, et al. Ustekinumab serum concentrations are associated with clinical outcomes in Crohn's disease a regional multi-center pilot study. Z Gastroenterol 2020;58:439-44.

[11] Hanzel J, Zdovc J, Kurent T, et al. Peak concentrations of ustekinumab after intravenous induction therapy identify patients with Crohn's disease likely to achieve endoscopic and biochemical remission. Clin Gastroenterol Hepatol 2021;19:111-8.

[12] Painchart C, Brabant S, Duveau N, et al. Ustekinumab serum trough levels may identify suboptimal responders to ustekinumab in Crohn's disease. Dig Dis Sci 2020;65:1445-52.

[13] Soufflet N, Boschetti G, Roblin X, et al. Concentrations of ustekinumab during induction therapy associate with remission in patients with Crohn's disease. Clin Gastroenterol Hepatol 2019;17: 2610-2. 